\title{
On Approximate Pure Nash Equilibria in Weighted Congestion Games with Polynomial Latencies
}

\author{
Ioannis Caragiannis
}

University of Patras \& CTI "Diophantus", Patras, Greece

caragian@ceid.upatras.gr

Angelo Fanelli

CNRS (UMR-6211), Caen, France

angelo.fanelli@unicaen.fr

\begin{abstract}
We consider the problem of the existence of natural improvement dynamics leading to approximate pure Nash equilibria, with a reasonable small approximation, and the problem of bounding the efficiency of such equilibria in the fundamental framework of weighted congestion game with polynomial latencies of degree at most $d \geq 1$.

In this work, by exploiting a simple technique, we firstly show that the game always admits a $d$-approximate potential function. This implies that every sequence of $d$-approximate improvement moves by the players always leads the game to a $d$-approximate pure Nash equilibrium. As a corollary, we also obtain that, under mild assumptions on the structure of the players' strategies, the game always admits a constant approximate potential function. Secondly, by using a simple potential function argument, we are able to show that in the game there always exists a $(d+\delta)$-approximate pure Nash equilibrium, with $\delta \in[0,1]$, whose cost is $2 /(1+\delta)$ times the cost of an optimal state.
\end{abstract}

2012 ACM Subject Classification Theory of computation $\rightarrow$ Algorithmic game theory; Theory of computation $\rightarrow$ Convergence and learning in games

Keywords and phrases Congestion games, approximate pure Nash equilibrium, potential functions, approximate price of stability

Digital Object Identifier 10.4230/LIPIcs.ICALP.2019.133

Category Track C: Foundations of Networks and Multi-Agent Systems: Models, Algorithms and Information Management

Funding Angelo Fanelli: ANR-14-CE24-0007-01 "CoCoRICo-CoDec"

\section{Introduction}

Among other solution concepts, the notion of pure Nash equilibrium plays a central role in Game Theory. Pure Nash equilibria in a game characterize situations in which no player has an incentive to unilaterally deviate from the current situation in order to achieve a higher payoff. Unfortunately, it is well known that there are games that do not have pure Nash equilibria. Furthermore, even in games where the existence of pure Nash equilibria is guaranteed, these equilibria could be very inefficient compared to solutions dictated by a central authority. Such negative results significantly question the importance of pure Nash equilibria as solution concepts that characterize the behavior of rational players.

One way to overcome the limitations of the non-existence and inefficiency of pure Nash equilibria is to consider a relaxation of the stability constraints. This relaxation leads to the concept of approximate pure Nash equilibrium. This concept characterizes situations where no player can significantly improve her payoff by unilaterally deviating from her current strategy. Approximate pure Nash equilibria can accommodate small modeling inaccuracies due to uncertainty (e.g., see the arguments in [5]), therefore they may be more desirable as 
solution concepts in practical decision-making settings. Beside mere existence and efficiency, approximate pure Nash equilibria are also an appealing alternative solution concept from a computational point of view $[2,4]$.

In this work, we investigate the existence and efficiency of approximate pure Nash equilibria in the context of the weighted congestion game. This game is a general framework which models situations in which a group of agents compete for the use of a set of shared resources. In the following, we introduce weighted congestion games and give a formal statement of the problems we address. We conclude this section with a discussion about the current literature and a detailed presentation of our contribution.

Weighted congestion games. In a weighted congestion game, players compete over a set of resources. Each player has a positive weight. Each resource incurs a latency to all players using it; this latency depends on the total weight (congestion) of the players that use the resource according to a resource-specific, non-negative, and non-decreasing latency function. Among a given set of strategies (over sets of resources), each player aims to select one selfishly, trying to minimize her individual total cost, i.e., the sum of the latencies on the resources in her strategy. Typical examples include weighted congestion games in networks, where the network links correspond to the resources and each player has alternative paths that connect two nodes as strategies. Now, let us describe the game more formally.

The WEIGHTED CONGESTION GAME with polynomial latencies of degree at most $d \in \mathbb{Z}^{\geq 1}$ is a collection of instances, denoted by $\operatorname{WCG}(d)$, of the form $G=$ $\left\langle N, E,\left(w_{i}\right)_{i \in N},\left(S_{i}\right)_{i \in N},\left(a_{e}, k_{e}\right)_{e \in E}\right\rangle$, where $N=\{1,2, \ldots,|N|\}$ is the set of players, $E=\{1,2, \ldots,|E|\}$ is the set of resources, $w_{i} \in \mathbb{R}^{>0}$ is the weight of player $i$, $S_{i} \subseteq 2^{E}$ is the set of strategies of player $i$ and $\left(a_{e}, k_{e}\right) \in \mathbb{R}^{>0} \times\{1,2, \ldots, d\}$ are the coefficient and the degree of resource $e \in E$ respectively, which encode the latency function $\ell_{e}: 2^{N} \mapsto \mathbb{R}^{\geq 0}$ associated with $e$, mapping every subset of players $P \subseteq N$ to the non-negative real $a_{e}\left(\sum_{j \in P} w_{j}\right)^{k_{e}}$.

The set of states of $G$ is denoted by $\mathcal{S}(G)=S_{1} \times S_{2} \times \ldots \times S_{|N|}$. For every state $\mathbf{s}$ we refer to its $i$-th component, that is the strategy played by player $i$ in $\mathbf{s}$, by $\mathbf{s}(i)$. For every state $\mathbf{s}$ and resource $e \in E$, we denote by $L_{e}(\mathbf{s})$ the set of players using resource $e$ in s, i.e., $L_{e}(\mathbf{s})=\{j \in N: e \in \mathbf{s}(i)\}$. We refer to the sum of the weights of all the players in $L_{e}(\mathbf{s})$ as the congestion of $e$ in $\mathbf{s}$. For every state $\mathbf{s} \in \mathcal{S}(G)$, the cost incurred by player $i \in N$ in $\mathbf{s}$ is $c_{i}(\mathbf{s})=\sum_{e \in \mathbf{s}(i)} \ell_{e}\left(L_{e}(\mathbf{s})\right)$, while the social cost of $\mathbf{s}$ is the weighted sum of the players' costs, i.e., $C(\mathbf{s})=\sum_{i \in N} w_{i} c_{i}(\mathbf{s})$. Notice that, by summing over the resources instead of the players, $C(\mathbf{s})$ can be rewritten as $C(\mathbf{s})=\sum_{e \in E} a_{e}\left(\sum_{j \in L_{e}(\mathbf{s})} w_{j}\right)^{k_{e}+1}$. Let $w_{\max }$ be the greatest weight in $G$, we say that $G$ is mildly congested if $\ell_{e}\left(L_{e}(\mathbf{s})\right) \geq\left(k_{e}+1\right) w_{\max }$, for every resource $e \in E$ and state $\mathbf{s} \in \mathcal{S}(G)$.

Preliminary definitions. We now introduce concepts that are necessary to formally state our problems and present our results.

Let us consider an instance $G=\left\langle N, E,\left(w_{i}\right)_{i \in N},\left(S_{i}\right)_{i \in N},\left(a_{e}, k_{e}\right)_{e \in E}\right\rangle$ of WCG $(d)$. For every state $\mathbf{s} \in \mathcal{S}(G)$ and every $s \in S_{i}$, we denote by $\left[\mathbf{s}_{-i}, s\right]$ the new state obtained from $\mathbf{s}$ by setting the $i$-th component, that is the strategy of $i$, to $s$ and keeping all the remaining components unchanged, i.e., $\left[\mathbf{s}_{-i}, s\right](i)=s$. The transition from $\mathbf{s}$ to $\left[\mathbf{s}_{-i}, s\right]$ is called a move of player $i$ from state $\mathbf{s}$. For $\alpha \geq 1$, we say that a transition from $\mathbf{s}$ to $\left[\mathbf{s}_{-i}, s\right]$ is an $\alpha$-improvement move for $i$ if $\alpha c_{i}\left(\left[\mathbf{s}_{-i}, s\right]\right) \leq c_{i}(\mathbf{s})$ (it is a strictly $\alpha$-improvement if $\left.\alpha c_{i}\left(\left[\mathbf{s}_{-i}, s\right]\right)<c_{i}(\mathbf{s})\right)$. For $\alpha \geq 1$, 
we say that a state-value function $\Gamma: \mathcal{S}(G) \mapsto \mathbb{R}^{\geq 0}$ is an $\alpha$-approximate potential function for $G$ if it strictly decreases at every strictly $\alpha$-improvement move; formally, $\Gamma\left(\left[\mathbf{s}_{-i}, s\right]\right)<\Gamma(\mathbf{s})$ whenever $\alpha c_{i}\left(\left[\mathbf{s}_{-i}, s\right]\right)<c_{i}(\mathbf{s})$. If $G$ admits an $\alpha$-approximate potential function $\Gamma$ then every sequence of strictly $\alpha$-improvement moves leads to a local optimum of $\Gamma$, that is a state in which no further strictly improvement move can be performed; such a state is called $\alpha$-approximate pure Nash equilibrium. Formally, for $\alpha \geq 1$, we say that a state $\mathbf{s} \in \mathcal{S}(G)$ is an $\alpha$-approximate pure Nash equilibrium if, for every player $i \in N$ and every strategy $s \in S_{i}$, we have $c_{i}(\mathbf{s}) \leq \alpha c_{i}\left(\left[\mathbf{s}_{-i}, s\right]\right)$. If $\alpha=1$ we simply refer to $\mathbf{s}$ as a pure Nash equilibrium rather than a 1-approximate pure Nash equilibrium. For $\alpha \geq 1$, we denote by $\mathcal{E}_{\alpha}(G) \subseteq \mathcal{S}(G)$ the set of all $\alpha$-approximate pure Nash equilibria of $G$. Every state $\mathbf{s} \in \mathcal{S}(G)$ minimizing the social cost is called a social optimum. We denote by $\mathcal{O P} \mathcal{T}(G)$ the set of social optima of $G$, i.e., $\mathcal{O P} \mathcal{T}(G)=\arg \min _{\mathbf{s} \in \mathcal{S}(G)} C(\mathbf{s})$. Let $\mathbf{o} \in \mathcal{O P} \mathcal{P}(G)$ be any social optimum of $G$, we define the $\alpha$-approximate price of stability of $G$ as $\operatorname{PoS}_{\alpha}(G)=\min _{\mathbf{e} \in \mathcal{E}_{\alpha}(G)} \frac{C(\mathbf{e})}{C(\mathbf{o})}$.

Problem statement. In this work we consider the problem of the existence of natural improvement dynamics leading to approximate pure Nash equilibria, with a reasonable small approximation, and the problem of bounding the efficiency of such equilibria in the fundamental framework of weighted congestion game with polynomial latencies of degree at most $d \geq 1$. We formally state such problems as follows.

(i) Existence of Convergent sequences of $\alpha$-Improvement moves. In this problem, given any instance $G$ of $\operatorname{WCG}(d)$, we seek for a reasonable small $\alpha \geq 1$ for which any sequence of $\alpha$-improvement moves in $G$ converges to an $\alpha$-approximate pure Nash equilibrium. This would be equivalent to say that $G$ admits an $\alpha$-approximate potential function, whose value decreases at every $\alpha$-improvement move and whose local optima coincide with $\alpha$-approximate pure Nash equilibria.

(ii) Bounding the APproximate Price of Stability. In this problem, given any instance $G$ of WCG $(d)$, which admits an $\alpha$-approximate pure Nash equilibrium, we aim at bounding the $\alpha$-approximate price of stability of $G$.

Related work. The unweighted congestion game (i.e., when all players have unit weight) has been widely studied in the literature. Rosenthal [16] proved that this game admits a 1-approximate potential function. This immediately implies that every sequence of 1improvement moves by the players leads the game to a pure Nash equilibrium. For the weighted congestion game, a 1-approximate potential function exists only when the latencies are linear or exponential $[10,13,15]$. For polynomial latencies (of constant maximum degree strictly higher than 1), pure Nash equilibria may not exist $[10,11,14]$. In general, for arbitrary latencies, the problem of deciding whether a given instance of weighted congestion game has a pure Nash equilibrium is NP-hard [9]. Caragiannis et al. [2] proved that every instance of weighted congestion game with polynomial latencies of degree at most $d$ admits a $d$ !-approximate potential function. This results has been subsequently improved by Hansknecht et al. [12]; they showed that every instance of weighted congestion game with polynomial latencies admits a $(d+1)$-approximate potential function. The potential function they proposed is a Rosenthal-like potential function. Roughly speaking, they obtained an approximate potential as follows. For each resource, they chose an appropriate fixed ordering of the players. Then, for each resource separately, they computed a discrete integral. Specifically, they sum up the latency of the resource after introducing the first player multiplied 
with the weight of the first player, the latency after introducing the first two players multiplied with the weight of the second player, and so on, i.e., $w_{1} \ell_{e}\left(\left\{w_{1}\right\}\right)+w_{2} \ell_{e}\left(\left\{w_{1}+w_{2}\right\}\right)+\ldots$. The potential obtained depends on the way the players have been initially ordered. The authors showed that, the potential function providing the best approximation for polynomial latencies, that is $d+1$, is the one obtained by ordering the players in non-decreasing order in terms of their weights, i.e., $w_{1} \leq w_{2} \leq \ldots$.

For the 1-approximate price of stability, for the unweighted game, there exists a bound of 1.577 for linear latencies $[8,3]$ and a bound of $\Theta(d)$ for polynomial latencies [6]. The 1-approximate price of stability for the weighted game with polynomial latencies has been recently investigated in [7]; they provided a lower bound of $\Omega(d / \log d)^{d+1}$, matching the upper bound in [1]. The authors also showed bounds to the $\alpha$-approximate price of stability. Specifically, they proved that for the weighted congestion game with polynomial latencies and weights ranging in $[1, W]$, there exists an $\alpha$-approximate pure Nash equilibrium, for any $\alpha$ in the range $\left[\frac{2(d+1) W}{2 W+d+1}, d+1\right]$, whose cost is $1+\left(\frac{d+1}{\alpha}-1\right) W$ the cost of any optimal state. Their proof exploits a potential function called Faulhaber's potential.

Our contribution. Concerning the first problem, we show (Theorem 3) that every instance of $\operatorname{WCG}(d)$ admits in general $d$-approximate potential functions. This implies that every sequence of $d$-approximate improvement moves by the players always leads the game to a $d$-approximate pure Nash equilibrium. This result is achieved by using the technique formalised in Theorem 2 and the class of state-value functions $\Phi_{\gamma}$ defined in Definition 1. Essentially, while Definition 1 provides a simple interesting class of candidate potential functions, Theorem 2 gives a local condition to each resource to determine the approximation guarantee achieved by a given state-value function. So, by exploiting Theorem 2, in Theorem 3 we are able to show that the class $\Phi_{\gamma}$ contains $d$-approximate potential functions and, more generally, $(d+\delta)$-approximate potential functions, for every $\delta \geq 0$. We remark that, our potential functions are substantially different from the potential function proposed in [12]. In fact, while the potential in [12] is obtained in a Rosenthal-like fashion, by ordering the players and summing their costs, by assuming that each player is affected only by the congestion caused by preceding players in the ordering, our potential, more simply, is obtained by a suitable scaling of the coefficients of the polynomials. As a matter of fact, our potentials, despite their simplicity, provide an approximation of $d$ instead of $d+1$; although it is worth noticing that, for very small degrees, the two approaches provide the same approximation guarantee. As a corollary of Theorem 3, we also show (Corollary 4) that the social optimum of an instance of $\operatorname{WCG}(d)$ is always a $(d+1)$-approximate pure Nash equilibrium, as it has already been observed in [7]. More importantly, Theorem 3 implies that, as state by Corollary 5 , every mildly congested instance of $\operatorname{WCG}(d)$ always admits a $\frac{\boldsymbol{e}}{\boldsymbol{e}-1}$-approximate potential function, where $\boldsymbol{e}$ is the Euler's number.

We also show that, the class of functions $\Phi_{\gamma}$ also serves as an essential tool to give a constant bound on the approximate price of stability. In fact, by exploiting $\Phi_{\gamma}$, we are able to show (Theorem 8) an upper bound of $2 /(1+\delta)$ for the $(d+\delta)$-approximate price of stability, for every $\delta \in[0,1]$. To prove this bound, we use the standard potential function argument. Specifically, we first give bounds (Lemma 6 and Lemma 7) relating the value of the $(d+\delta)$-approximate potential function for a given state to the social cost of that state; if we then perform a sequence of $(d+\delta)$-improvement moves starting from an optimal state, the potential does not increase, and hence we can bound the cost of any $(d+\delta)$-approximate pure Nash equilibrium that we reach. Notice that our bound does not depend on the range of the players' weights and significantly improves the bound provided in [7], by making use of a different and simpler potential function. 
Roadmap. We begin with the definition of a class of state-value functions in Section 2. In Section 3 we first present a simple technique to bound the approximation guarantee of a given state-value function, subsequently we show that some elements of the class introduced in Section 2 provide a good approximation. In Section 4 we present the bound on the approximate price of stability. Finally, in section 5 we present a couple of technical lemmas.

\section{A class of state-value functions}

In this section we define a class of functions mapping every state of the game to a non-negative real number. This class of functions will be exploited in the subsequent sections.

- Definition 1. Let $G=\left\langle N, E,\left(w_{i}\right)_{i \in N},\left(S_{i}\right)_{i \in N},\left(a_{e}, k_{e}\right)_{e \in E}\right\rangle$ be an instance of WCG $(d)$. For every resource $e \in E$ and every $\gamma=\left(\gamma_{e}\right)_{e \in E}$, we define

$$
\Phi_{\gamma}(\mathbf{s})=\sum_{e \in E} a_{e} \Psi_{e}^{\gamma_{e}}\left(L_{e}(\mathbf{s})\right)
$$

where, for every nonempty subset of players $P \subseteq N$, we have

$$
\Psi_{e}^{\gamma_{e}}(P)=\frac{\gamma_{e}}{k_{e}+1}\left(\sum_{j \in P} w_{j}\right)^{k_{e}+1}+\left(1-\frac{\gamma_{e}}{k_{e}+1}\right) \sum_{j \in P} w_{j}^{k_{e}+1} .
$$

\section{Approximate potential functions}

The main result of this section is stated by Theorem 3, where we show the existence of good approximate potential functions. Before showing this result, in Theorem 2 we illustrate our tool to define an approximate potential function. Such tool gives a local condition to each resource to determine the approximation guarantee of a given state-value function. We conclude this section with two corollaries, the first (Corollary 4) showing that the social optimum of an instance of $\operatorname{WCG}(d)$ is always a $(d+1)$-approximate pure Nash equilibrium, the second (Corollary 5) showing that, under mild conditions, the game always admits a constant approximate potential function.

- Theorem 2. Let $G=\left\langle N, E,\left(w_{i}\right)_{i \in N},\left(S_{i}\right)_{i \in N},\left(a_{e}, k_{e}\right)_{e \in E}\right\rangle$ be an instance of WCG $(d)$. Let $\Gamma: \mathcal{S}(G) \mapsto \mathbb{R}^{>0}$ be a state-value function such that $\Gamma(\mathbf{s})=\sum_{e \in E} a_{e} \Gamma_{e}\left(L_{e}(\mathbf{s})\right)$, where $\Gamma_{e}: 2^{N} \mapsto \mathbb{R}^{>0}$. If, for every resource $e \in E$, every non-empty subset of players $P \subseteq N$ and every player $i \in P$, there exist $\lambda_{e}, v_{e} \in \mathbb{R}^{>0}$, with $\lambda_{e} \leq v_{e}$, such that

$$
\frac{w_{i} \ell_{e}(P)}{a_{e}\left(\Gamma_{e}(P)-\Gamma_{e}(P \backslash\{i\})\right)} \in\left[\lambda_{e}, v_{e}\right]
$$

then $\Gamma$ is a $\left(\frac{v}{\lambda}\right)$-approximate potential function for $G$, where $v=\max _{e \in E} v_{e}$ and $\lambda=$ $\min _{e \in E} \lambda_{e}$.

Proof. Let us consider a state $\mathbf{s} \in \mathcal{S}(G)$ and a player $i$. Let us assume that $i$ can perform an $\frac{v}{\lambda}$-improvement move by replacing strategy $\mathbf{s}(i)$ with $s \neq \mathbf{s}(i)$, i.e., $\frac{v}{\lambda} c_{i}\left(\left[\mathbf{s}_{-i}, s\right]\right)<c_{i}(\mathbf{s})$. In order to prove the claim we need to show that $\Gamma\left(\left[\mathbf{s}_{-i}, s\right]\right)<\Gamma(\mathbf{s})$. To this aim, let us bound the expression $\Gamma\left(\left[\mathbf{s}_{-i}, s\right]\right)-\Gamma(\mathbf{s})$. We have, 


$$
\begin{aligned}
\Gamma\left(\left[\mathbf{s}_{-i}, s\right]\right)-\Gamma(\mathbf{s})= & \sum_{e \in E} a_{e} \Gamma_{e}\left(L_{e}\left(\left[\mathbf{s}_{-i}, s\right]\right)\right)-\sum_{e \in E} a_{e} \Gamma_{e}\left(L_{e}(\mathbf{s})\right) \\
= & \sum_{e \in E} a_{e}\left[\Gamma_{e}\left(L_{e}\left(\left[\mathbf{s}_{-i}, s\right]\right)\right)-\Gamma_{e}\left(L_{e}(\mathbf{s})\right)\right] \\
= & \sum_{e \in s \backslash \mathbf{s}(i)} a_{e}\left[\Gamma_{e}\left(L_{e}\left(\left[\mathbf{s}_{-i}, s\right]\right)\right)-\Gamma_{e}\left(L_{e}(\mathbf{s})\right)\right] \\
& \quad-\sum_{e \in \mathbf{s}(i) \backslash s} a_{e}\left[\Gamma_{e}\left(L_{e}(\mathbf{s})\right)-\Gamma_{e}\left(L_{e}\left(\left[\mathbf{s}_{-i}, s\right]\right)\right)\right] \\
= & \sum_{e \in s} a_{e}\left[\Gamma_{e}\left(L_{e}\left(\left[\mathbf{s}_{-i}, s\right]\right)\right)-\Gamma_{e}\left(L_{e}(\mathbf{s})\right)\right] \\
& \quad-\sum_{e \in \mathbf{s}(i)} a_{e}\left[\Gamma_{e}\left(L_{e}(\mathbf{s})\right)-\Gamma_{e}\left(L_{e}\left(\left[\mathbf{s}_{-i}, s\right]\right)\right)\right] \\
\leq & \sum_{e \in s} \frac{1}{\lambda_{e}} w_{i} \ell_{e}\left(L_{e}\left(\left[\mathbf{s}_{-i}, s\right]\right)\right)-\sum_{e \in \mathbf{s}(i)} \frac{1}{v_{e}} w_{i} \ell_{e}\left(L_{e}(\mathbf{s})\right) \\
\leq & \frac{1}{\lambda} \sum_{e \in s} w_{i} \ell_{e}\left(L_{e}\left(\left[\mathbf{s}_{-i}, s\right]\right)\right)-\frac{1}{v} \sum_{e \in \mathbf{s}(i)} w_{i} \ell_{e}\left(L_{e}(\mathbf{s})\right) \\
= & \frac{w_{i}}{v}\left(\frac{v}{\lambda} c_{i}\left(\left[\mathbf{s}_{-i}, s\right]\right)-c_{i}(\mathbf{s})\right)
\end{aligned}
$$

where (2) follows from the fact that for every $e \in \mathbf{s}(i) \cap s$ the variation of $\Gamma_{e}$ is null since $L_{e}\left(\left[\mathbf{s}_{-i}, s\right]\right)=L_{e}(\mathbf{s})$, (3) from (1), (4) from the definition of $\lambda$ and $v$ and (5) from the definition of cost.

From (5) we obtain that, if $\frac{v}{\lambda} c_{i}\left(\left[\mathbf{s}_{-i}, s\right]\right)<c_{i}(\mathbf{s})$ then $\Gamma\left(\left[\mathbf{s}_{-i}, s\right]\right)<\Gamma(\mathbf{s})$, from which the claim follows.

We are ready to present the main result of this section.

- Theorem 3. Let $G=\left\langle N, E,\left(w_{i}\right)_{i \in N},\left(S_{i}\right)_{i \in N},\left(a_{e}, k_{e}\right)_{e \in E}\right\rangle$ be an instance of $\mathrm{WCG}(d)$. It holds that

(a) if $\gamma_{e}=1$, for every $e \in E$, then $\Phi_{\gamma}$ is a $\rho(G)$-approximate potential function for $G$, where

$$
\rho(G)=\max _{e \in E} \sup _{x>0} \frac{(1+x)^{k_{e}}}{\frac{1}{k_{e}+1}(1+x)^{k_{e}+1}+\frac{k_{e}}{k_{e}+1}-\frac{1}{k_{e}+1} x^{k_{e}+1}} \leq d ;
$$

(b) if $\gamma_{e}=k_{e}$, for every $e \in E$, then $\Phi_{\gamma}$ is a d-approximate potential function for $G$;

(c) if $\gamma_{e}=k_{e}+\delta$, for every $e \in E$ and $\delta \geq 0$, then $\Phi_{\gamma}$ is a $(d+\delta)$-approximate potential function for $G$.

Proof. We prove the claim using Theorem 2. Therefore, for every resource $e \in E$, every non-empty subset of players $P \subseteq N$ and every player $i \in P$, we bound the ratio

$$
\frac{w_{i} \ell_{e}(P)}{a_{e}\left(\Psi_{e}^{\gamma_{e}}(P)-\Psi_{e}^{\gamma_{e}}(P \backslash\{i\})\right)} .
$$

Let us explicitly rewrite the numerator and denominator of the previous expression. We distinguish between the cases $|P|=\{i\}$ and $|P| \geq 2$. 
Let us fist assume $|P|=\{i\}$. In this case, for $w_{i} \ell_{e}(P)$ we get

$$
w_{i} \ell_{e}(P)=w_{i} a_{e}\left(w_{i}\right)^{k_{e}}=a_{e} w_{i}^{k_{e}+1} .
$$

On the other hand, for the expression $\Psi_{e}^{\gamma_{e}}(P)-\Psi_{e}^{\gamma_{e}}(P \backslash\{i\})$, using Definition 1, we have

$$
\Psi_{e}^{\gamma_{e}}(P)-\Psi_{e}^{\gamma_{e}}(P \backslash\{i\})=\left[\frac{\gamma_{e}}{k_{e}+1}\left(w_{j}\right)^{k_{e}+1}+\left(1-\frac{\gamma_{e}}{k_{e}+1}\right) w_{j}^{k_{e}+1}\right]-0=w_{j}^{k_{e}+1} .
$$

Therefore, by combining (8) and (9), we conclude that, for $|P|=\{i\}$, for the ratio (7) we have

$$
\frac{w_{i} \ell_{e}(P)}{a_{e}\left(\Psi_{e}^{\gamma_{e}}(P)-\Psi_{e}^{\gamma_{e}}(P \backslash\{i\})\right)}=\frac{a_{e} w_{i}^{k_{e}+1}}{a_{e} w_{i}^{k_{e}+1}}=1 .
$$

Not let us assume that $|P| \geq 2$. In this case, for $w_{i} \ell_{e}(P)$ we get

$$
\begin{aligned}
w_{i} \ell_{e}(P) & =w_{i} a_{e}\left(\sum_{j \in P} w_{j}\right)^{k_{e}}=w_{i} a_{e}\left(w_{i}+\sum_{j \in P \backslash\{i\}} w_{j}\right)^{k_{e}} \\
& =a_{e} w_{i}^{k_{e}+1}\left(1+\lambda_{i}(P)\right)^{k_{e}} .
\end{aligned}
$$

Now, let us focus on the expression $\Psi_{e}^{\gamma_{e}}(P)-\Psi_{e}^{\gamma_{e}}(P \backslash\{i\})$. For every $i \in P$, let $\lambda_{i}(P)=$ $\frac{1}{w_{i}} \sum_{j \in P \backslash\{i\}} w_{j}$. Using Definition 1, we have

$$
\begin{aligned}
& \Psi_{e}^{\gamma_{e}}(P)-\Psi_{e}^{\gamma_{e}}(P \backslash\{i\}) \\
= & \frac{\gamma_{e}}{k_{e}+1}\left(\sum_{j \in P} w_{j}\right)^{k_{e}+1}+\left(1-\frac{\gamma_{e}}{k_{e}+1}\right) \sum_{j \in P} w_{j}^{k_{e}+1} \\
& -\frac{\gamma_{e}}{k_{e}+1}\left(\sum_{j \in P \backslash\{i\}} w_{j}\right)^{k_{e}+1}-\left(1-\frac{\gamma_{e}}{k_{e}+1}\right) \sum_{j \in P \backslash\{i\}} w_{j}^{k_{e}+1} \\
= & \frac{\gamma_{e}}{k_{e}+1}\left(\sum_{j \in P} w_{j}\right)^{k_{e}+1}+\left(1-\frac{\gamma_{e}}{k_{e}+1}\right) w_{i}^{k_{e}+1}-\frac{\gamma_{e}}{k_{e}+1}\left(\sum_{j \in P \backslash\{i\}} w_{j}\right)^{k_{e}+1} \\
= & \frac{\gamma_{e}}{k_{e}+1}\left(w_{i}+\sum_{j \in P \backslash\{i\}} w_{j}\right)^{k_{e}+1}+\left(1-\frac{\gamma_{e}}{k_{e}+1}\right) w_{i}^{k_{e}+1}-\frac{\gamma_{e}}{k_{e}+1}\left(\sum_{j \in P \backslash\{i\}} w_{j}\right)^{k_{e}+1} \\
= & \frac{\gamma_{e}}{k_{e}+1} w_{i}^{k_{e}+1}\left(1+\lambda_{i}(P)\right)^{k_{e}+1}+\left(1-\frac{\gamma_{e}}{k_{e}+1}\right) w_{i}^{k_{e}+1}-\frac{\gamma_{e}}{k_{e}+1} w_{i}^{k_{e}+1} \lambda_{i}(P)^{k_{e}+1} \\
= & w_{i}^{k_{e}+1}\left[\frac{\gamma_{e}}{k_{e}+1}\left(1+\lambda_{i}(P)\right)^{k_{e}+1}+\left(1-\frac{\gamma_{e}}{k_{e}+1}\right)-\frac{\gamma_{e}}{k_{e}+1} \lambda_{i}(P)^{k_{e}+1}\right]
\end{aligned}
$$

Therefore, by combining (11) and (12), we conclude that, for $|P| \geq 2$, for the expression (7) we have 


$$
\begin{aligned}
& \frac{w_{i} \ell_{e}(P)}{a_{e}\left(\Psi_{e}^{\gamma_{e}}(P)-\Psi_{e}^{\gamma_{e}}(P \backslash\{i\})\right)} \\
= & \frac{a_{e} w_{i}^{k_{e}+1}\left(1+\lambda_{i}(P)\right)^{k_{e}}}{a_{e} w_{i}^{k_{e}+1}\left[\frac{\gamma_{e}}{k_{e}+1}\left(1+\lambda_{i}(P)\right)^{k_{e}+1}+\left(1-\frac{\gamma_{e}}{k_{e}+1}\right)-\frac{\gamma_{e}}{k_{e}+1} \lambda_{i}(P)^{k_{e}+1}\right]} \\
= & \frac{\left(1+\lambda_{i}(P)\right)^{k_{e}}}{\frac{\gamma_{e}}{k_{e}+1}\left(1+\lambda_{i}(P)\right)^{k_{e}+1}+\left(1-\frac{\gamma_{e}}{k_{e}+1}\right)-\frac{\gamma_{e}}{k_{e}+1} \lambda_{i}(P)^{k_{e}+1}} .
\end{aligned}
$$

Now we apply Lemma 9 to (13), by setting $x=\lambda_{i}(P), h=k_{e}$ and $\beta=\gamma_{e}$. If $\gamma_{e}=1$, we obtain

$$
\frac{w_{i} \ell_{e}(P)}{a_{e}\left(\Psi_{e}^{\gamma_{e}}(P)-\Psi_{e}^{\gamma_{e}}(P \backslash\{i\})\right)} \in\left[1, k_{e}\right] .
$$

If $\gamma_{e}=k_{e}$, we have

$$
\frac{w_{i} \ell_{e}(P)}{a_{e}\left(\Psi_{e}^{\gamma_{e}}(P)-\Psi_{e}^{\gamma_{e}}(P \backslash\{i\})\right)} \in\left[\frac{1}{k_{e}}, 1\right] .
$$

Finally, if $\gamma_{e}=k_{e}+\delta$, with $\delta \geq 0$, we have

$$
\frac{w_{i} \ell_{e}(P)}{a_{e}\left(\Psi_{e}^{\gamma_{e}}(P)-\Psi_{e}^{\gamma_{e}}(P \backslash\{i\})\right)} \in\left[\frac{1}{k_{e}+\delta}, 1\right] .
$$

Claim (a) follows by combining (10), (13) and (14), and by applying Theorem 2. Claims (b) and (c) follow by combining (10), (15) and (16), and by applying Theorem 2.

- Corollary 4. Let $G=\left\langle N, E,\left(w_{i}\right)_{i \in N},\left(S_{i}\right)_{i \in N},\left(a_{e}, k_{e}\right)_{e \in E}\right\rangle$ be an instance of $\operatorname{WCG}(d)$. Any social optimum of $G$ is a $(d+1)$-approximate pure Nash equilibrium.

Proof. Let us consider the function $\Phi_{\gamma}(\mathbf{s})=\sum_{e \in E} \Psi_{e}^{\gamma_{e}}\left(L_{e}(\mathbf{s})\right)$ (defined in Definition 1), with $\gamma_{e}=k_{e}+1$. The claim follows from observing that $\Phi_{\gamma}(\mathbf{s})=C(\mathbf{s})$ and from the fact that, by Theorem $3(\mathrm{c}), \Phi_{\gamma}$ is also a $(d+1)$-approximate potential function.

- Corollary 5. Let $G=\left\langle N, E,\left(w_{i}\right)_{i \in N},\left(S_{i}\right)_{i \in N},\left(a_{e}, k_{e}\right)_{e \in E}\right\rangle$ be a mildly congested instance of $\mathrm{WCG}(d)$. If $\gamma_{e}=1$, for every $e \in E$, then $\Phi_{\gamma}$ is a $\frac{e}{e-1}$-approximate potential function for $G$, where $\boldsymbol{e}$ is the Euler's number.

Proof Sketch. Since $G$ is mildly congested, we have $\ell_{e}\left(L_{e}(\mathbf{s})\right) \geq\left(k_{e}+1\right) w_{\max }$, for every resource $e \in E$ and state $\mathbf{s} \in \mathcal{S}(G)$, where $w_{\max }$ denotes the greatest weight in $G$. Under this condition, we can restrict the proof of Theorem 3 to the case in which the generic subset of player $P$ is such that $\sum_{j \in P} w_{j} \geq\left(k_{e}+1\right) w_{\max }$. With this condition in place, we have that, for every $i \in P, \lambda_{i}(P)$ is at least $k_{e}$. In fact,

$$
\lambda_{i}(P)=\frac{1}{w_{i}}\left(\sum_{j \in P} w_{j}-w_{i}\right) \geq \frac{1}{w_{i}}\left(\left(k_{e}+1\right) w_{\max }-w_{i}\right) \geq \frac{w_{\max }}{w_{i}} k_{e} \geq k_{e} .
$$

By using the previous inequality, it is easy to prove that the expression (13) gets always values in the range $\left[1, \frac{e}{e-1}\right]$, for every $k_{e} \geq 1$. The claim follows by applying Theorem 2 . 


\section{Approximate price of stability}

In this section we show an upper bound on the $\alpha$-approximate price of stability, for $\alpha \in$ $[d, d+1]$. This bound is stated by Theorem 8 , whose proof is based on Lemma 6 and Lemma 7 presented below.

- Lemma 6. Let $G=\left\langle N, E,\left(w_{i}\right)_{i \in N},\left(S_{i}\right)_{i \in N},\left(a_{e}, k_{e}\right)_{e \in E}\right\rangle$ be an instance of $\operatorname{WCG}(d)$. Let $\gamma_{e}=k_{e}+\delta$ and $\delta \in[0,1]$, for every resource $e \in E$. For every resource $e \in E$ and every non-empty subset of players $P \subseteq N$, we have

$$
\Psi_{e}^{\gamma_{e}}(P) \leq\left(\sum_{j \in P} w_{j}\right)^{k_{e}+1} \leq \frac{k_{e}+1}{k_{e}+\delta} \Psi_{e}^{\gamma_{e}}(P) .
$$

Proof. We have

$$
\begin{aligned}
\frac{\left(\sum_{j \in P} w_{j}\right)^{k_{e}+1}}{\Psi_{e}^{\gamma_{e}}(P)} & =\frac{\left(\sum_{j \in P} w_{j}\right)^{k_{e}+1}}{\frac{k_{e}+\delta}{k_{e}+1}\left(\sum_{j \in P} w_{j}\right)^{k_{e}+1}+\left(1-\frac{k_{e}+\delta}{k_{e}+1}\right) \sum_{j \in P} w_{j}^{k_{e}+1}} \\
& \in\left[1, \frac{k_{e}+1}{k_{e}+\delta}\right) .
\end{aligned}
$$

where (17) follows from Definition 1 and the definition of $\gamma_{e}$, and (18) follows by applying Lemma 10, where we set $x=\left(\sum_{j \in P} w_{j}\right)^{k_{e}+1}, y=\sum_{j \in P} w_{j}^{k_{e}+1}$ and $\beta=\frac{k_{e}+1}{k_{e}+\delta}$.

In the following lemma, we give bounds relating the value of the approximate potential function for a given state to the social cost of that state.

- Lemma 7. Let $G=\left\langle N, E,\left(w_{i}\right)_{i \in N},\left(S_{i}\right)_{i \in N},\left(a_{e}, k_{e}\right)_{e \in E}\right\rangle$ be an instance of $\operatorname{WCG}(d)$. Let $\gamma=\left(\gamma_{e}\right)_{e \in E}$, where $\gamma_{e}=k_{e}+\delta$ and $\delta \in[0,1]$. For every state $\mathbf{s} \in \mathcal{S}(G)$ we have

$$
\Phi_{\gamma}(\mathbf{s}) \leq C(\mathbf{s}) \leq \frac{2}{1+\delta} \Phi_{\gamma}(\mathbf{s}) .
$$

Proof. Let $E=\left\{e_{1}, e_{2}, \ldots, e_{m}\right\}$. Let us bound the ratio

$$
\frac{C(\mathbf{s})}{\Phi_{\gamma}(\mathbf{s})}=\frac{\sum_{t=1}^{m} a_{e_{t}}\left(\sum_{j \in L_{e_{t}}(\mathbf{s})} w_{j}\right)^{k_{e_{t}}+1}}{\sum_{t=1}^{m} a_{e_{t}} \Psi_{e_{t}}^{\gamma_{e_{t}}}\left(L_{e_{t}}(\mathbf{s})\right)} .
$$

In order to bound $C(\mathbf{s}) / \Phi_{\gamma}(\mathbf{s})$, we consider the ratio between the $t$-th term in the numerator and the $t$-th term in the denominator, for every $t \in[1, m]$, that is

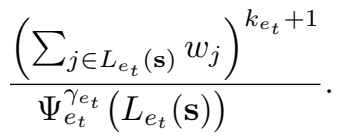

From Lemma $6,(20)$ gets values in the interval $\left[1, \frac{k_{e_{t}}+1}{k_{e_{t}}+\delta}\right]$. We get that the smallest ratio is 1 while the greatest one is $\max _{t} \frac{k_{e_{t}}+1}{k_{e_{t}}+\delta} \leq \frac{2}{1+\delta}$. It follows that, $C(\mathbf{s}) / \Phi_{\gamma}(\mathbf{s})$ is at least 1 and at most $\frac{2}{1+\delta}$, from which the claim follows.

Theorem 8. Let $G=\left\langle N, E,\left(w_{i}\right)_{i \in N},\left(S_{i}\right)_{i \in N},\left(a_{e}, k_{e}\right)_{e \in E}\right\rangle$ be an instance of $\operatorname{WCG}(d)$. Then $\operatorname{PoS}_{d+\delta}(G) \leq \frac{2}{1+\delta}$, for every $\delta \in[0,1]$. 
Proof. Let $\Phi_{\gamma}$ be the function with $\gamma=\left(\gamma_{e}\right)_{e \in E}$, where $\gamma_{e}=k_{e}+\delta$. Let $\mathbf{o} \in \mathcal{O P T}(G)$ be a social optimum. Let us consider any sequence of $(d+\delta)$-improvement moves starting from o. From Theorem 3(c) we know that this sequence converges to a state which is a $(d+\delta)$-approximate pure Nash equilibrium which we denote by e. Moreover, along this sequence of moves, $\Phi_{\gamma}$ is not increasing. Hence,

$$
\Phi_{\gamma}(\mathbf{e}) \leq \Phi_{\gamma}(\mathbf{o}) .
$$

By applying Lemma 7 repeatedly to both $\mathbf{o}$ and $\mathbf{e}$ we obtain

$$
C(\mathbf{e}) \leq \frac{2}{1+\delta} \Phi_{\gamma}(\mathbf{e}) \leq \frac{2}{1+\delta} \Phi_{\gamma}(\mathbf{o}) \leq \frac{2}{1+\delta} C(\mathbf{o}),
$$

where the second inequality follows from (21). From which the claim follows.

\section{Technical lemmas}

In this section we present two technical lemmas. Lemma 9 is used in the proof of Theorem 3 , while Lemma 10 is used in the proof of Lemma 6.

- Lemma 9. For every $x \in \mathbb{R}^{>0}, h \in \mathbb{Z}^{\geq 1}$ and $\beta \in \mathbb{R}^{\geq 1}$, we have

$$
\frac{(1+x)^{h}}{\beta \frac{1}{h+1}(1+x)^{h+1}+\left(1-\beta \frac{1}{h+1}\right)-\beta \frac{1}{h+1} x^{h+1}} \in \begin{cases}{\left[\frac{1}{\beta}, \frac{h}{\beta}\right]} & \text { if } \beta \in[1, h] \\ {\left[\frac{1}{\beta}, 1\right]} & \text { if } \beta \geq h .\end{cases}
$$

Proof. We have

$$
\begin{aligned}
& \frac{(1+x)^{h}}{\beta \frac{1}{h+1}(1+x)^{h+1}+\left(1-\beta \frac{1}{h+1}\right)-\beta \frac{1}{h+1} x^{h+1}} \\
= & \frac{\sum_{t=0}^{h}\left(\begin{array}{c}
h \\
t
\end{array}\right) x^{t}}{\beta \frac{1}{h+1} \sum_{t=0}^{h+1}\left(\begin{array}{c}
h+1 \\
t
\end{array}\right) x^{t}+\left(1-\beta \frac{1}{h+1}\right)-\beta \frac{1}{h+1} x^{h+1}} \\
= & \frac{1+\sum_{t=1}^{h}\left(\begin{array}{c}
h \\
t
\end{array}\right) x^{t}}{1+\beta \frac{1}{h+1} \sum_{t=1}^{h+1}\left(\begin{array}{c}
h+1 \\
t
\end{array}\right) x^{t}-\beta \frac{1}{h+1} x^{h+1}} \\
= & \frac{1+\sum_{t=1}^{h}\left(\begin{array}{c}
h \\
t
\end{array}\right) x^{t}}{1+\sum_{t=1}^{h} \beta \frac{1}{h+1}\left(\begin{array}{c}
h+1 \\
t
\end{array}\right) x^{t}} \\
= & \frac{1+\sum_{t=1}^{h}\left(\begin{array}{c}
h \\
t
\end{array}\right) x^{t}}{1+\sum_{t=1}^{h} \beta \frac{1}{h+1} \frac{h+1}{h+1-t}\left(\begin{array}{c}
h \\
t
\end{array}\right) x^{t}}=\frac{1+\sum_{t=1}^{h}\left(\begin{array}{c}
h \\
t
\end{array}\right) x^{t}}{1+\sum_{t=1}^{h} \beta \frac{1}{h+1-t}\left(\begin{array}{c}
h \\
t
\end{array}\right) x^{t}} \\
= & \frac{1 \cdot x^{0}+\left(\begin{array}{c}
h \\
1
\end{array}\right) x^{1}+\quad\left(\begin{array}{c}
h \\
2
\end{array}\right) x^{2}+\ldots+\left(\begin{array}{c}
h \\
h
\end{array}\right) x^{h}}{1 \cdot x^{0}+\beta \frac{1}{h}\left(\begin{array}{l}
h \\
1
\end{array}\right) x^{1}+\beta \frac{1}{h-1}\left(\begin{array}{c}
h \\
2
\end{array}\right) x^{2}+\ldots+\beta\left(\begin{array}{c}
h \\
h
\end{array}\right) x^{h}},
\end{aligned}
$$

where (22) holds because

$$
\left(\begin{array}{c}
h+1 \\
t
\end{array}\right)=\frac{\frac{(h+1) !}{t !(h+1-t) !}}{\frac{h !}{t !(h-t) !}}\left(\begin{array}{l}
h \\
t
\end{array}\right)=\frac{(h+1) !}{t !(h+1-t) !} \frac{t !(h-t) !}{h !}\left(\begin{array}{l}
h \\
t
\end{array}\right)=\frac{h+1}{h+1-t}\left(\begin{array}{l}
h \\
t
\end{array}\right) .
$$

In order to bound (23), for every $t \in[0, h]$ we consider the ratio between the coefficient of the term $x^{t}$ in the numerator and the coefficient of the same term in the denominator. For $t=0$ the ratio is 1 , while for $t \in[1, h]$ the ratio is $\frac{h+1-t}{\beta}$. For the case $\beta \in[1, h]$, we get that the smallest ratio is $1 / \beta$ while the greatest is $h / \beta$. It follows that, when $\beta \in[1, h]$, the expression in (23) is at least $1 / \beta$ and at most $h / \beta$. For the case $\beta \geq h$, we obtain that the smallest is $1 / \beta$ while the greatest ratio is $1 \mathrm{df}$. Therefore, when $\beta \geq h$, the expression in (23) is at least $1 / \beta$ and at most 1 . From which the claim follows. 
- Lemma 10. For every $x, y, \beta \in \mathbb{R}^{>0}$ such that $\beta \geq 1$ and $y \leq x$, we have

$$
\frac{x}{\frac{1}{\beta} x+\left(1-\frac{1}{\beta}\right) y} \in[1, \beta] \text {. }
$$

Proof. We have

$$
\frac{x}{\frac{1}{\beta} x+\left(1-\frac{1}{\beta}\right) y}=\frac{1}{\frac{1}{\beta}+\left(1-\frac{1}{\beta}\right) \frac{y}{x}} \geq \frac{1}{\frac{1}{\beta}+\left(1-\frac{1}{\beta}\right)}=1,
$$

where the last inequality is due to the fact that $y / x \leq 1$.

$$
\frac{x}{\frac{1}{\beta} x+\left(1-\frac{1}{\beta}\right) y}=\frac{1}{\frac{1}{\beta}+\left(1-\frac{1}{\beta}\right) \frac{y}{x}}<\frac{1}{\frac{1}{\beta}}=\beta,
$$

where the last inequality is due to the fact that $y / x>0$.

\section{References}

1 Sebastian Aland, Dominic Dumrauf, Martin Gairing, Burkhard Monien, and Florian Schoppmann. Exact price of anarchy for polynomial congestion games. SIAM Journal on Computing, 40(5):1211-1233, 2011.

2 Ioannis Caragiannis, Angelo Fanelli, Nick Gravin, and Alexander Skopalik. Approximate pure Nash equilibria in weighted congestion games: Existence, efficient computation, and structure. ACM Transactions on Economics and Computation, 3(1):2:1-2:32, 2015.

3 Ioannis Caragiannis, Michele Flammini, Christos Kaklamanis, Panagiotis Kanellopoulos, and Luca Moscardelli. Tight bounds for selfish and greedy load balancing. Algorithmica, 61(3):606-637, 2011.

4 Steve Chien and Alistair Sinclair. Convergence to approximate Nash equilibria in congestion games. Games and Economic Behavior, 71(2):315-327, 2011.

5 Nicolas Christin, Jens Grossklags, and John Chuang. Near Rationality and Competitive Equilibria in Networked Systems. In Proceedings of the ACM SIGCOMM Workshop on Practice and Theory of Incentives in Networked Systems (PINS), pages 213-219, 2004.

6 George Christodoulou and Martin Gairing. Price of stability in polynomial congestion games. ACM Transactions on Economics and Computation, 4(2):10:1-10:17, 2016.

7 George Christodoulou, Martin Gairing, Yiannis Giannakopoulos, and Paul G. Spirakis. The Price of Stability of Weighted Congestion Games. In Proceedings of the 45th International Colloquium on Automata, Languages, and Programming (ICALP), pages 150:1-150:16, 2018.

8 George Christodoulou and Elias Koutsoupias. On the price of anarchy and stability of correlated equilibria of linear congestion games. In Proceedings of the 13th Annual European Symposium on Algorithms (ESA), pages 59-70, 2005.

9 Juliane Dunkel and Andreas S. Schulz. On the complexity of pure-strategy Nash equilibria in congestion and local-effect games. Mathematics of Operations Research, 33(4):851-868, 2008

10 Dimitris Fotakis, Spyros C. Kontogiannis, and Paul G. Spirakis. Selfish unsplittable flows. Theoretical Computer Science, 348(2-3):226-239, 2005.

11 Michel X. Goemans, Vahab S. Mirrokni, and Adrian Vetta. Sink equilibria and convergence. In Proceedings of the 46th Annual IEEE Symposium on Foundations of Computer Science (FOCS), pages 142-154, 2005.

12 Christoph Hansknecht, Max Klimm, and Alexander Skopalik. Approximate pure Nash equilibria in weighted congestion games. In Approximation, Randomization, and Combinatorial Optimization. Algorithms and Techniques (APPROX/RANDOM), pages 242-257, 2014.

13 Tobias Harks and Max Klimm. On the existence of pure Nash equilibria in weighted congestion games. In Proceedings of the 37th International Colloquium on Automata, Languages and Programming (ICALP), Part I, pages 79-89, 2010. 
133:12 On Approximate Pure Nash Equilibria in Weighted Congestion Games

14 Lavy Libman and Ariel Orda. Atomic resource sharing in noncooperative networks. Telecommunication Systems, 17(4):385-409, 2001.

15 Panagiota N. Panagopoulou and Paul G. Spirakis. Algorithms for pure Nash equilibria in weighted congestion games. ACM Journal of Experimental Algorithmics, 11, 2006.

16 Robert W. Rosenthal. A class of games possessing pure-strategy Nash equilibria. International Journal of Game Theory, 2:65-67, 1973. 